\title{
Androgen deficiency in older men
}

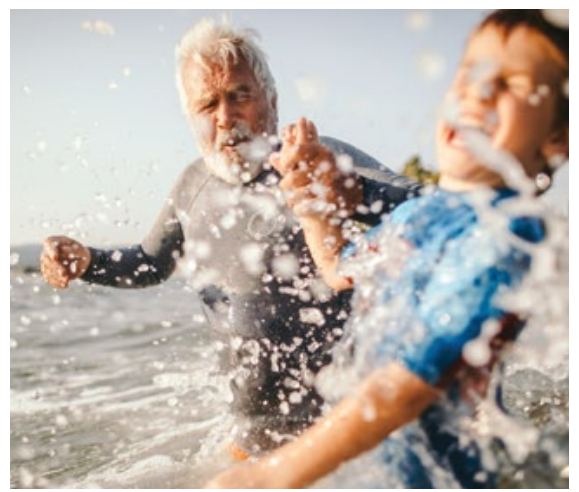

\section{Mathis Grossmann}

\section{Background}

Older men, especially those who are overweight or obese and have comorbidities, not uncommonly present with non-specific androgen deficiencylike symptoms, such as low energy and sexual dysfunction, and modestly lowered serum testosterone relative to reference ranges based on healthy young men.

\section{Objective}

The aim of this paper is to describe the clinical approach to men who present with non-specific androgen deficiencylike symptoms.

\section{Discussion}

Most men who present with non-specific androgen deficiency-like symptoms do not have organic hypogonadism due to pituitary or testicular disease, but instead have functional gonadal axis suppression due to ill health. Lifestyle measures especially weight loss, optimisation of comorbidities and cessation of offending medications - can improve symptoms and increase serum testosterone; this should be the first-line approach. Recent randomised controlled trials (RCTs) have reported modest benefits of testosterone treatment in stringently selected older men. However, the true long-term risks and benefits of testosterone treatment in such men are not known. Further study is required before testosterone treatment can be routinely recommended for indications other than organic hypogonadism.

\section{Organic versus functional hypogonadism}

For older men with suspected androgen deficiency, making the clinical distinction between organic and functional hypogonadism is important (Table 1). This is because organic hypogonadism can be due to potentially serious pathology, such as a pituitary lesion. Moreover, the treatment approach differs (Figure 1).

\section{Organic hypogonadism}

Organic hypogonadism due to medical disease of the hypothalamic-pituitarytesticular (HPT) axis, such as a pituitary tumour or Klinefelter syndrome, is an important diagnosis that should not be overlooked. It is important to consider organic hypogonadism in a man of any age presenting with otherwise unexplained clinical features such as psychosexual complaints, osteoporosis, anaemia or sarcopenia. ${ }^{1}$ Organic hypogonadism can occur at any age, with some older men presenting with primary hypogonadism due to age-related testicular failure, evidenced by high gonadotrophin levels, reduced testicular response to human chorionic gonadotropin, and reduced Leydig cell mass. ${ }^{2}$

There is good evidence that organic hypogonadism is underdiagnosed. Australian studies show that less than half of men with Klinefelter syndrome are diagnosed in their lifetimes. ${ }^{3}$ In men with organic hypogonadism, testosterone replacement has marked benefits on sexual function, energy, haemoglobin, bone density and body composition, and should be considered irrespective of age. However, testosterone does not improve fertility but instead can suppress spermatogenesis. ${ }^{4}$

\section{Functional hypogonadism}

Many older men - especially those who are overweight or obese and have chronic diseases such as diabetes, depression, liver or renal disease - present with non-specific, androgen deficiency-like symptoms such as fatigue, low energy, hot flushes and sexual dysfunction (low libido and/or erectile dysfunction). They may also have clinical features overlapping with those of organic hypogonadism, such as central adiposity, sarcopaenia and reduced bone mineral density. Such men commonly have modest reductions in their circulating testosterone concentrations, typically approximately 6-10 nmol/L, relative to reference ranges based on healthy young men. ${ }^{2}$

Testosterone concentrations decline gradually and modestly during male ageing - a marked difference to the abrupt and severe decline that occurs in female menopause. It is also worth noting that ageing and hypogonadism have overlapping features. These observations have led to a concept of so called 'late-onset' hypogonadism. The estimated prevalence of late-onset hypogonadism, defined as the coexistence of androgen deficiency-like features and lowered circulating testosterone in the absence of organic HPT axis pathology, 
is estimated to be approximately $2-5 \%$ in community-dwelling older men. ${ }^{5}$ However, the extent to which lowered testosterone concentrations contribute to the ageing male phenotype is not known. It is also possible that lowered testosterone is primarily a consequence of functional HPT suppression due to ill health (Figure 2). It is clear that low testosterone is, at the very least, a sensitive marker of poor health. Indeed, recent Australian research has shown that the age-related decline in testosterone may in part be due to the accumulation of age-related comorbidities and weight gain, rather than solely due to age in itself. ${ }^{6}$ These studies suggest that the age-related decline in testosterone may be preventable by management of health and lifestyle factors.

\section{Work-up of older men presenting with features suggestive of androgen deficiency}

\section{Clinical approach}

The clinical work-up is primarily focused on excluding a diagnosis of organic hypogonadism. This is because:

- organic hypogonadism may be the consequence of an underlying pathology

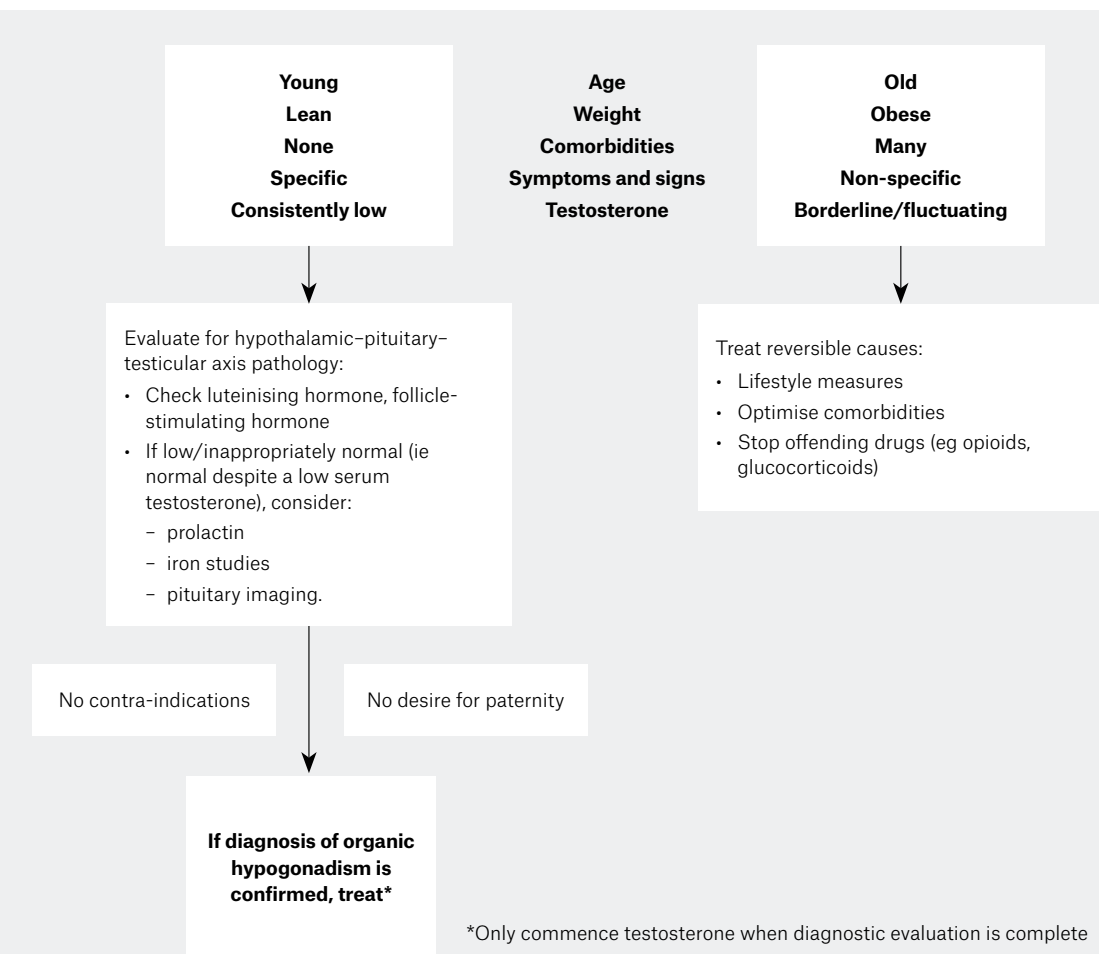

Figure 1. The clinical approach to possible hypogonadism, based on underlying patient characteristics. Men with features in the upper left box are more likely to have organic hypogonadism and should be evaluated for hypothalamic-pituitary-testicular (HPT) axis pathology prior to consideration of testosterone replacement. Men with features in the upper right box are more likely to have functional hypogonadism, and detailed evaluation for HPT axis pathology is of low yield. In these men, the focus should be on treatment of reversible causes of functional HPT axis suppression.

Table 1. Organic hypogonadism versus functional hypogonadism

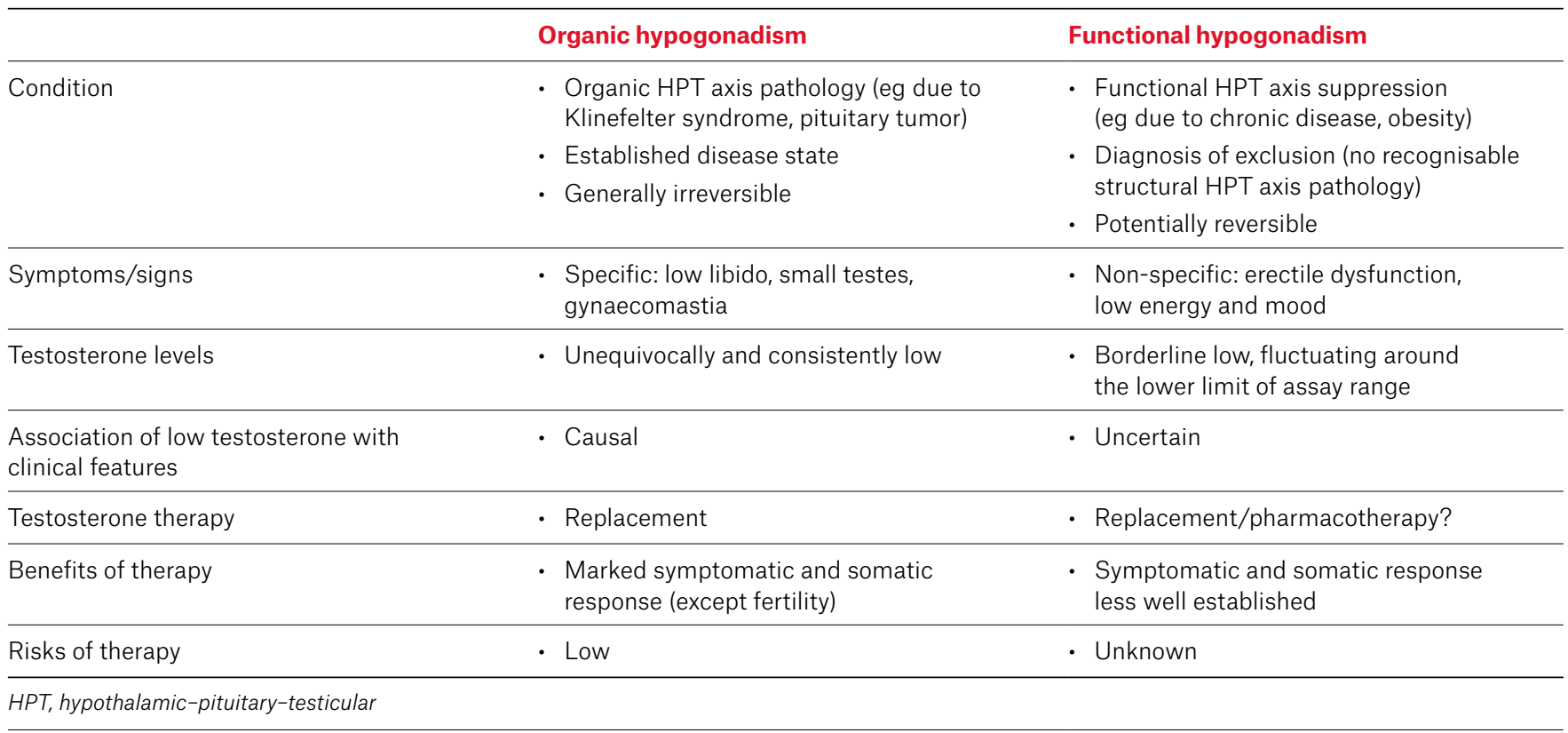




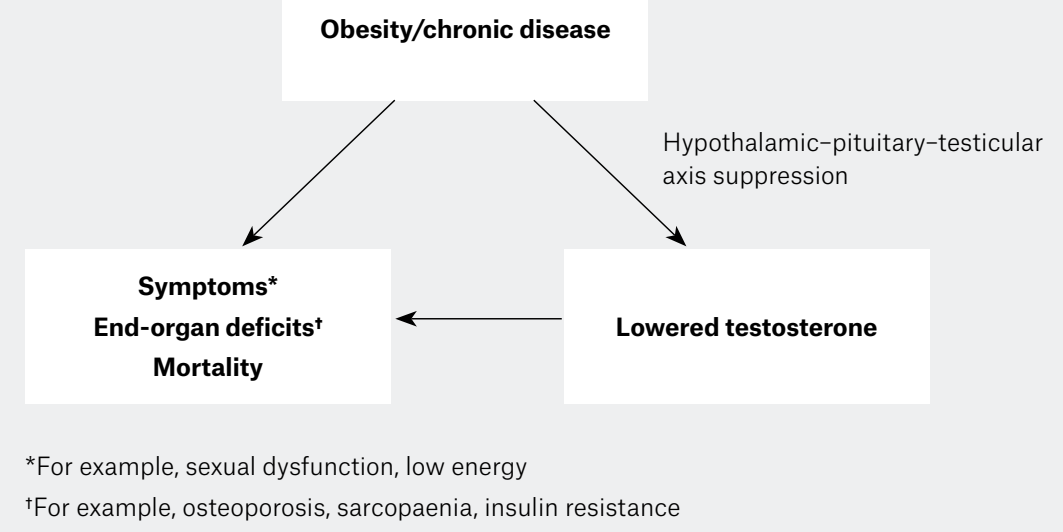

Figure 2. The interplay between obesity/chronic disease, androgen deficiency-like features and circulating testosterone. Obesity and chronic disease can lead to nonspecific hypothalamic-pituitary-testicular (HPT) axis suppression, which is functional and potentially reversible. The extent to which low testosterone contributes to androgen deficiency-like features is not well defined.

Table 2. Effects of testosterone treatment in randomised controlled trials of older men*

\begin{tabular}{|c|c|c|}
\hline Concern & Testosterone treatment effect & Targeted treatment \\
\hline \multirow[t]{2}{*}{ Sexual } & $\begin{array}{l}\text { Modest improvement in most domains } \\
\text { of sexual function (if testosterone } \\
<9.5-12 \mathrm{nmol} / \mathrm{L} \text { ) }\end{array}$ & $\begin{array}{l}\text { Phosphodiesterase type } \\
5 \text { inhibitor for treatment } \\
\text { of erectile dysfunction }\end{array}$ \\
\hline & $\begin{array}{l}\text { In most RCTs, effect on libido more marked/ } \\
\text { consistent than effect on erectile function }\end{array}$ & \\
\hline \multirow[t]{2}{*}{ Muscle } & $\begin{array}{l}1.6-2.7 \mathrm{~kg} \text { increase in mass, and increase } \\
\text { in strength }\end{array}$ & Exercise \\
\hline & $\begin{array}{l}\text { Modest improvements in some measures } \\
\text { of muscle performance (eg small increase } \\
\text { in walking distance) }\end{array}$ & \\
\hline Fat & $1.6-2 \mathrm{~kg}$ decrease & Weight loss \\
\hline \multirow[t]{2}{*}{$\begin{array}{l}\text { Glucose } \\
\text { metabolism }\end{array}$} & $\begin{array}{l}\text { Modest improvement in insulin resistance } \\
\text { in some RCTs }\end{array}$ & $\begin{array}{l}\text { Lifestyle, antidiabetic } \\
\text { medications }\end{array}$ \\
\hline & No effect on $\mathrm{HbA1c}$ & \\
\hline \multirow[t]{2}{*}{ Bone } & $\begin{array}{l}2-7 \% \text { increase in lumbar spine bone } \\
\text { mineral density }\end{array}$ & Antiresorptives \\
\hline & $\begin{array}{l}\text { RCTs have not been large enough to assess } \\
\text { effects on fractures }\end{array}$ & \\
\hline Mood & $\begin{array}{l}\text { Slight improvement in mood/depressive } \\
\text { symptoms }\end{array}$ & $\begin{array}{l}\text { Counselling, } \\
\text { antidepressants }\end{array}$ \\
\hline Cognition & No effect on cognition or memory & $\begin{array}{l}\text { Cholinesterase } \\
\text { inhibitors }\end{array}$ \\
\hline
\end{tabular}

*In these RCTs, older men with organic hypogonadism were excluded.

$H b A 1 c$, glycated haemoglobin; RCT, randomised controlled trial; BMI, body mass index that should not be overlooked, such as

a pituitary tumour

- the approach to management differs (Figure 1).

Given that hypogonadism is a clinical diagnosis supported by consistent biochemical findings, older men who present with features suggestive of androgen deficiency should have a thorough history and physical examination to determine the degree of clinically significant androgen deficiency. ${ }^{1}$ Clinical assessment should focus on eliciting the more specific features of androgen deficiency, such as gynaecomastia, recent loss of body hair and decreasing testicular volume. Features such as fatigue, low libido and reduced muscle bulk are non-specific and can be caused by almost any chronic disease. ${ }^{2}$ Initial assessment should include the identification of comorbidities that may confound the clinical picture or represent potentially reversible causes, such as obesity, depression, uncontrolled sleep apnoea or medications such as opioids or glucocorticoids. Clues to underlying organic aetiology, such as signs of pituitary dysfunction or mass effect, should not be missed. Surreptitious anabolic steroid use should always be considered.

\section{Laboratory testing and pituitary imaging}

The clinical impression of androgen deficiency should be confirmed by measuring serum total testosterone using an accurate and reliable assay, with blood drawn in the morning in the fasted state. ${ }^{1}$ This is important given the circadian rhythmicity of testosterone (with lower concentrations later in the day), and because food intake can decrease testosterone by up to $25 \%$. Men should be clinically stable, as any intercurrent illness can suppress testosterone. Given day-to-day variability of testosterone (up to $30 \%$ ), a diagnosis of androgen deficiency should never be based on a single low testosterone result. By contrast, a total testosterone of $>12 \mathrm{nmol} / \mathrm{L}$ is generally consistent with eugonadism and does not need to be repeated, unless there is suspicion of evolving androgen deficiency. Although liquid 
chromatography/mass spectrometrybased testosterone assays are considered gold standard, their current availability for routine clinical use is limited.

Testosterone immunoassays performed by accredited laboratories are generally reliable to confirm eugonadism, although they may have some imprecision at the lower end of the male reference range. ${ }^{7}$ Results have to be interpreted in the clinical context. Different assay platforms have different reference ranges; there is some variability in measurements across different platforms. While a discussion about free testosterone (usually calculated from total testosterone, sex hormonebinding globulin and albumin) is beyond the scope, its measurement is generally of limited practical value. ${ }^{1}$ There are no validated reference ranges for free testosterone, especially in older men.

The majority of older men with lowered testosterone will have low-tonormal gonadotropin concentrations due to functional hypothalamic-pituitary inhibition from chronic disease, including obesity (Figure 2). ${ }^{8}$ Evaluation for underlying organic HPT axis pathology is commonly of low yield and should be individualised. In men with non-elevated gonadotropins, the probability of organic hypothalamic-pituitary pathology is inversely related to body mass index, age, number of comorbidities and testosterone concentrations. In the absence of clinical suspicion of pituitary disease, biochemical work-up can be limited to measuring prolactin and, in men $<65$ years, iron studies to exclude haemochromatosis. The testosterone cut-off below which pituitary imaging is necessary is not well defined, but imaging should be considered in men with a total testosterone of $<5.2 \mathrm{nmol} / \mathrm{L}$ and low-to-normal gonadotrophins, even in the absence of clinical suspicion of hypothalamic pituitary disease. ${ }^{9}$

\section{Management of functional hypogonadism}

Men with organic hypogonadism of any age should be considered for testosterone replacement, which has been discussed elsewhere. ${ }^{4}$

\section{Lifestyle and optimisation of comorbidities}

The majority of older men will have functional, non-destructive suppression of the HPT axis and present with non-specific symptoms and borderline low testosterone concentrations fluctuating around the lower end (6-10 $\mathrm{nmol} / \mathrm{L})$ of the reference range derived from healthy young men. ${ }^{2}$

Priority should be given to the management of reversible causes, most importantly the implementation of lifestyle measures to achieve weight loss, optimisation of comorbidities (eg depression, sleep apnoea or glycaemic control in diabetes) and, where possible, removal of offending medications (eg opioids, glucocorticoids; Figure 1). This strategy can safely raise testosterone concentrations and have other health benefits. Explaining this to affected men can increase their motivation to engage with these measures.

Obesity is the strongest risk factor for low testosterone, even greater than the effects of age, in part because obesity blunts the age-related rise in luteinising hormone that can compensate for testicular dysfunction occurring in some older men. ${ }^{8}$ Consistent with this, successful weight loss, whether by diet or surgery, can lead to substantial increases in testosterone in obese men. The increase in testosterone is proportional to the amount of weight lost: $10 \%$ weight loss increases testosterone by 2-3 nmol/L, whereas profound weight loss after bariatric surgery in morbidly obese men can raise testosterone by more than $10 \mathrm{nmol} / \mathrm{L}^{2}$

Almost any acute or chronic disease can mimic or confound clinical features of androgen deficiency, and the condition should be optimised. This will improve non-specific symptoms and can lead to an increase in testosterone concentrations. For example, in observational studies, implementation of continuous positive airway pressure for sleep apnoea, improvement of glycaemic control in men with diabetes and opioid or glucocorticoid cessation led to increases in testosterone concentrations of approximately 3-5 nmol/L. ${ }^{2}$

\section{Testosterone therapy}

For some men, measures to reverse functional hypogonadism may be unsuccessful, either because implementation is not feasible (eg cessation of opioids) or they are not achieved or maintained (eg weight loss). For other men, symptoms may persist despite successful implementation of these measures. For some men, low testosterone may contribute to fatigue or poor motivation and thereby reduce their ability to initiate healthy lifestyle measures. This raises the question of whether testosterone therapy should be considered in selected circumstances, and what the benefits and risks of testosterone treatment are. These have been reviewed elsewhere, ${ }^{10}$ with a brief summary discussed here. Of note, effective first-line therapies that target individual clinical domains are available (Table 2).

\section{Possible benefits of testosterone therapy}

The largest clinical trial of testosterone treatment in older men, the Testosterone Trials, randomised 790 men aged $\geq 65$ years with a baseline testosterone $<9.54 \mathrm{nmol} / \mathrm{L}$ (mean of at least two measures) and at least one symptom or sign consistent with hypogonadism (decreased libido, difficulty walking or low vitality), but no evidence of organic hypogonadism, to testosterone or placebo for 12 months. ${ }^{11}$ Testosterone treatment resulted in modest benefits in sexual function and slightly improved mood and depressive symptoms, but did not improve cognitive function. Concomitant sub-studies showed improvements in volumetric bone mineral density and anaemia. ${ }^{10,11}$ Other smaller studies have reported modest improvements in body composition (approximately $2 \mathrm{~kg}$ gain in muscle mass and $2 \mathrm{~kg}$ loss of fat mass) and modest improvements in insulin resistance but, in men with diabetes, no improvements in glycaemic control (Table 2). ${ }^{12}$

Whether testosterone treatment reduces important clinical endpoints such as falls, fractures or mortality in older men is not known. This is because existing RCTs of testosterone therapy in older men have been of relatively small size and of 
short duration. Some RCTs have included men who were either asymptomatic or did not have confirmed low testosterone. Therefore, more evidence is needed. For example, T4DM, a large Australian RCT, examines whether testosterone treatment prevents diabetes in high risk men. ${ }^{13}$ Results are expected in 2020.

\section{Possible risks of testosterone therapy}

Older men with significant comorbidities may be at higher risk of adverse outcomes from testosterone therapy when compared with younger, otherwise healthy men receiving testosterone replacement for organic hypogonadism. Trials to date have been underpowered to provide definitive outcome data regarding prostate and cardiovascular events, and long-term risks are unknown. It has been estimated that an RCT enrolling several thousand men over 3-5 years would be needed to define true long-term risks; the largest RCT to date enrolled 790 men over 12 months. ${ }^{11}$

Regarding prostate events, the data have been reassuring. Despite the fact that androgen deprivation therapy is an effective treatment for established prostate cancer, clinical studies to date have not shown that testosterone treatment increases the risk of developing prostate cancer. ${ }^{4}$ Prostate monitoring during testosterone therapy may, however, lead to overdiagnosis of pre-existing, clinically insignificant prostate cancer. ${ }^{9}$

The current evidence regarding testosterone treatment and cardiovascular outcomes is contradictory and inconclusive. Some studies suggest increased risks, but these results have not been supported by the majority of studies. ${ }^{10}$

\section{Should testosterone treatment be considered for men without organic hypogonadism?}

Given gaps in the current evidence base, this is controversial. ${ }^{14}$ While Australian guidelines ${ }^{1}$ recommend against testosterone treatment for older men without organic hypogonadism, US Endocrine Society guidelines ${ }^{9}$ give an option for testosterone therapy as a shared decision between clinicians and carefully selected individual patients. ${ }^{14}$
If treatment is considered, men with functional hypogonadism should be informed about the absence of high-level evidence regarding long-term benefits and risks. Clear, patient-specific goals should be identified, and treatment should be stopped if these goals are not achieved. Monitoring for adverse events should follow consensus recommendations. ${ }^{4}$

\section{Conclusions}

Men with organic hypogonadism should be identified and considered for testosterone replacement. More research is needed to clarify the role for testosterone therapy in other settings. In most older men, low testosterone is a marker of poor health and should prompt a holistic approach with a focus on lifestyle measures and optimisation of comorbidities. Successful weight loss, combined with optimisation of comorbidities, can be sufficient to improve symptoms and increase testosterone concentrations, and will lead to other important health benefits for such men.

\section{Authors}

Mathis Grossmann MD, PhD, FRACP, Professor of Medicine, Department of Medicine, Austin Health, University of Melbourne; Head of Men's Health, Department of Endocrinology, Austin Health, Melbourne, Vic. mathisg@unimelb.edu.au Competing interests: None.

Funding: MG reports grants from Bayer Pharma, grants from Novartis, grants from Weight Watchers, grants from Lilly, personal fees from Besins, outside the submitted work.

Provenance and peer review: Commissioned, externally peer reviewed.

\section{References}

1. Yeap BB, Grossmann M, McLachlan RI, et al. Endocrine society of Australia position statement on male hypogonadism (part 1): Assessment and indications for testosterone therapy. Med J Aust 2016;205(4):173-78.

2. Grossmann M, Matsumoto AM. A perspective on middle-aged and older men with functional hypogonadism: Focus on holistic management. J Clin Endocrinol Metab 2017;102(3):1067-75. doi: 10.1210/jc.2016-3580.

3. Herlihy AS, Halliday JL, Cock ML, McLachlan RI. The prevalence and diagnosis rates of Klinefelter syndrome: An Australian comparison. Med J Aust 2011;194(1):24-28. doi: 10.5694/j.1326-5377.2011. tb04141.x.

4. Yeap BB, Grossmann M, McLachlan RI, et al. Endocrine society of Australia position statement on male hypogonadism (part 2): Treatment and therapeutic considerations. Med J Aust 2016;205(5):228-31. doi: 10.5694/mja16.00448.
5. Wu FC, Tajar A, Beynon JM, et al. Identification of late-onset hypogonadism in middle-aged and elderly men. N Engl J Med 2010;363(2):123-35. doi: 10.1056/NEJMoa0911101.

6. Sartorius G, Spasevska S, Idan A, et al. Serum testosterone, dihydrotestosterone and estradiol concentrations in older men self-reporting very good health: The healthy man study. Clin Endocrinol (Oxf) 2012;77(5):755-63. doi: 10.1111/j.1365-2265.2012.04432x.

7. Sikaris K, McLachlan RI, Kazlauskas R, de Kretser D, Holden CA, Handelsman DJ. Reproductive hormone reference intervals for healthy fertile young men: Evaluation of automated platform assays. J Clin Endocrinol Metab 2005;90(11):5928-36. doi: 10.1210/ jc.2005-0962.

8. Grossmann M. Hypogonadism and male obesity: Focus on unresolved questions. Clin Endocrinol (Oxf) 2018;89(1):11-21. doi: 10.1111/cen.13723.

9. Bhasin S, Brito JP, Cunningham GR, et al. Testosterone therapy in men with hypogonadism: An endocrine society clinical practice guideline. J Clin Endocrinol Metab 2018;103(5):1715-44. doi: 10.1210/jc.2018-00229.

10. Yeap BB, Page ST, Grossmann M. Testosterone treatment in older men: Clinical implications and unresolved questions from the testosterone trials. Lancet Diabetes Endocrinol 2018;6(8):659-72. doi: 10.1016/S2213-8587(17)30416-3.

11. Snyder PJ, Bhasin S, Cunningham GR, et al. Effects of testosterone treatment in older men. N Engl J Med 2016;374(7):611-24. doi: 10.1056/ NEJMoa1506119.

12. Grossmann M, Hoermann R, Wittert G, Yeap BB. Effects of testosterone treatment on glucose metabolism and symptoms in men with type 2 diabetes and the metabolic syndrome: A systematic review and meta-analysis of randomized controlled clinical trials. Clin Endocrinol (Oxf) 2015;83(3):344-51. doi: 10.1111/ cen.12664.

13. Wittert G, Atlantis E, Allan C, et al. Testosterone therapy to prevent type 2 diabetes mellitus in at-risk men (T4DM): Design and implementation of a double-blind randomised controlled trial. Diabetes Obes Metab 2018;21(4):772-80. doi: 10.1111/dom.13601.

14. Yeap BB, Wu FCW. Clinical practice update on testosterone therapy for male hypogonadism: Contrasting perspectives to optimize care. Clin Endocrinol (Oxf) 2019;90(1):56-5. doi: 10.1111/ cen.13888. 